\title{
DEFORMACIONES LITERARIAS: EMBRIOLOGÍA, GENEALOGÍA, Y CIENCIA FICCIÓN EN EL INFORME CABRERA DE JOSÉ “PEPE” LIBOY
}

\author{
POR \\ Emily A. Maguire \\ Northwestern University
}

Nada es lo que aparenta ser en El informe Cabrera (2009), la primera novela del escritor puertorriqueño José "Pepe" Liboy (1964). A primera vista, el libro se presenta como la carta de un padre a su hijo, una historia epistolar escrita para ser leída después de su muerte. Sin embargo, este hijo no ha nacido todavía, y no nacerá hasta (tal vez) cien años después de la muerte de su padre. Estamos frente a un ambiente de ciencia ficción, en un Puerto Rico futuro (y futurista) en que las familias que padecen de problemas genéticos evitan la posibilidad de malformaciones congénitas a través de la embriología, y donde los niños son planeados y "nacidos" en un centro de embriología en las montañas de la isla. Dentro de este escenario futurista, el narrador Manuel Molina (quien se parece a Liboy mismo) le escribe a su hijo para darle la historia de su procedencia: la vida de sus padres, sus experiencias formativas, la manera en que su concepción fue planeada y la ciencia embriológica que ayudó a que naciera.

La información más importante del Informe, sin embargo, no es esta historia biológica familiar; a la vez que narra el proceso de "crear" a su hijo, el padre/narrador relata otra historia genealógica: la de la evolución del mismo texto del Informe, el cual identifica como "[...] una especie de novela de ciencia ficción histórica, con algo de tremendismo biográfico [...]" (12). En la serie de cartas, notas, y otros textos que compone el "informe", Molina elabora una lista de los precursores de su novela, haciendo así una "genealogía" de la ciencia ficción puertorriqueña. Este canon literario resulta ser tan intencionalmente fabricado como el hijo del narrador, aunque el proceso de creación literaria opera en sentido contrario al proceso biológico: si el padre busca a una muchacha que pueda ser la madre ideal de su hijo futuro, el escritor recurre a la historia literaria de la isla en busca de textos que pueda reconocer o identificar como miembros de este género. El corpus de ciencia ficción puertorriqueña que perfila incluye escritores finiseculares nunca antes identificados como autores de ciencia ficción y novelistas y poetas contemporáneos del propio Liboy como Rafael Acevedo (1960), Aravind Adyanthaya (1965) y Yara Liceaga (1972). Este "informe" de la creación de un 
hijo biológico se vuelve igualmente una meditación sobre la creación -y la herencialiteraria y una exploración de los límites de un género literario.

La participación en comunidades literarias alternativas no es nada nuevo para Pepe Liboy. Aunque El Informe Cabrera es su primera novela, cuando ésta se publicó el escritor ya era un cuentista conocido, con un libro de cuentos (Cada vez te despides mejor, 2003) y una lista de otros cuentos que habían aparecido en varias antologías de literatura caribeña contemporánea. ${ }^{1}$ Según Juan Carlos Quintero Herencia, Liboy forma parte de un grupo de escritores jóvenes que han rechazado las formas dominantes de la escritura isleña para crear una comunidad literaria basada en la otredad y en el desencuentro con los discursos y las prácticas del escenario mediático de la literatura puertorriqueña. Juan Duchesne Winter nombra este grupo (que incluye a los escritores Yara Liceaga y Eduardo Lalo, entre otros) "los raros", y plantea que su escritura desafía el status quo literario puertorriqueño (31). Duchesne Winter diagnostica la "rareza" en la literatura puertorriqueña como una estrategia de sobrevivencia que responde tanto a la condición colonial de la isla como a la crisis (económica y social) que experimenta hoy en día. ${ }^{2}$ Para Duchesne Winter, la escritura de los raros "[...] pone en juego las bases de la representación misma y hace de la dificultad de comprensión inmediata, es decir, de la opacidad, un arma estética y política [...]" (33), creando así una escritura contestataria que resiste las presiones de la cultura burguesa (literaria) dominante.

El Informe Cabrera demuestra varias de las características que Duchesne Winter identifica como típicas de la escritura "rara". La construcción polifónica del texto de Liboy, que exhibe una intertextualidad densa y constante (hace referencia a un grupo extenso de novelistas, poetas, críticos e historiadores tanto locales como internacionales) produce una sensación de la susodicha opacidad. Otra técnica que Duchesne Winter reconoce en la escritura de los raros es lo que nombra "lo real múltiple", una forma que mezcla, incorpora o colapsa elementos realistas con elementos de lo fantástico para producir "[...] una articulación compenetrada de múltiples realidades y zonas de la experiencia donde nada deja de ser real" (36). La temática de la novela de Liboy -un hijo será engendrado en un laboratorio en el centro de una isla caribeña años después de la muerte de su padre biológico- podría tal vez ofrecer un ejemplo de esta técnica narrativa, ya que mezcla elementos de la vida del escritor y hechos de la historia literaria isleña con elementos puramente ficticios. A pesar de que el narrador es identificado

1 Ver Los nuevos caníbales: antología de la más reciente cuentística del Caribe hispano; El rostro y la máscara y Ma(h)ab(l)ar. Liboy también ha publicado cuentos y poemas en las revistas Filo de juego, Tríptico, y La Habana Elegante.

2 Para un resumen de los problemas económicos y sociales de Puerto Rico, ver Lizette Álvarez, "Economy and Crime Spur New Puerto Rican Exodus". The New York Times 8 February 2014. < http://www.nytimes. com/2014/02/09/us/economy-and-crime-spur-new-puerto-rican-exodus.html>. 
como Manuel Molina, hay varios momentos en el texto cuando parece que el narrador es Pepe Liboy mismo. ${ }^{3}$

Sin embargo, cabe destacar que Liboy identifica su texto explícitamente como ciencia ficción, un gesto intencional que diferencia su proyecto de las técnicas narrativas del género "fantástico" y hasta de lo meramente "raro". ¿Cuál es la función de este gesto autoreflexivo? ¿Por qué insiste Liboy (y, a través de él, el narrador) en conectar su novela con el género de la ciencia ficción? Christine Gledhill, crítica de cine, observa que "[...] genre is first and foremost a boundary phenomenon" (221), una práctica que puede servir más para subrayar diferencias o cambios en un modo textual que para enfatizar semejanzas textuales. Para Gledhill, el valor del género (literario, cinematográfico) como concepto yace precisamente en lo que se revela cuando un texto "rompe" con las reglas establecidas de un género o cuando se cambia la definición de qué constituye un género particular: "Genre analysis tells us not just about kinds of films but about the cultural work of producing and knowing them" (222). El estudio de un género literario, entonces, ofrece una manera de conectar un texto particular con su contexto. El hecho de que Liboy identifica El Informe Cabrera como ciencia ficción es importante no sólo por lo que nos puede decir sobre las fronteras literarias (genéricas) que Liboy perfila sino también por lo que le (nos) ofrece la ciencia ficción dentro del contexto literario puertorriqueño.

Carl Freedman observa que la ciencia ficción es el género más cercano al realismo histórico, ya que los dos demuestran una conciencia del desarrollo histórico. Para Freedman, la ciencia ficción hasta ofrece un "mejor" vehículo para tratar la historia que la novela histórica misma, ya que está libre - hasta cierto punto- del peso del pasado: "[Science fiction's] primary orientation [...] is toward the future; it is thus capable of engaging the matter of historicity without the same kind of post-1848 ideological baggage that the historical novelist carries" (58). La distancia temporal narrativa que ofrece la ciencia ficción le puede ser particularmente útil al narrador de El Informe Cabrera (y a Liboy mismo). No es casual, entonces, que el narrador de El Informe identifique su texto (o este compendio de textos) como ciencia ficción. Las características temporales del género - esta mirada hacia el futuro desde un presente que pronto será pasado- le dan las herramientas para indagar en los problemas de la historia puertorriqueña pero desde la posición más distante -más ecuánime- de un posible futuro. Sugiero que identificar su texto como ciencia ficción es también destacarlo (y destacarse) de lo que podría ser identificado como un realismo sencillo, es hacer hincapié en su rareza, en

3 La mezcla de lo real con lo ficticio comienza con la tapa del libro, lo cual anuncia el título del libro en letras de estilo de una película de horror. Al trasfondo hay una vista aérea de lo que podría ser una hacienda. En primer plano aparece una foto de la cabeza de Liboy mismo, rodeado de un aura de luz como si fuera un dios mirando la escena desde el cielo. Esta yuxtaposición produce el efecto estético de una revista pulp pero con toques de realidad. 
su diferencia. ${ }^{4} \mathrm{Al}$ caracterizar su texto como una obra de "ciencia ficción histórica", Liboy subraya lo que separa esta narrativa de otras narrativas, lo que podríamos llamar las "deformaciones" del texto.

En El Informe la ciencia ficción es una técnica que altera a la vez que es alterado. La ciencia ficción es un género que rompe con las condiciones miméticas del realismo, ya que sigue la lógica de un ambiente realista a la vez que introduce un elemento de lo que Darko Suvin llama "cognitive estrangement" (extrañamiento cognitivo 372), un componente que contiene su propia lógica científica pero que va más allá de las fronteras de un escenario realista. Seo-Young Chu explica, sin embargo, que tanto la ciencia ficción como el realismo operan según el mimetismo: “[...] what most people call 'science fiction' is actually a high-intensity variety of realism, one that requires astronomical levels of energy to accomplish its representational task insofar as its referents [...] elaborately defy straightforward representation" (7). Liboy ofrece este género como una especie de salida para la narrativa puertorriqueña, una ruta de escape que permite la expresión de lo que no podría ser comunicado con un realismo menos "intenso" (o menos "raro"). Al trazar un canon de la ciencia ficción puertorriqueña, plantea que esta ruta no es radicalmente nueva sino que ya tiene una tradición dentro de la isla.

La ciencia ficción en la novela de Liboy puede ser categorizada como una "deformación”, sin embargo, es sólo una alteración en una narrativa que opera a través de muchas distorsiones y alteraciones -sean genéticas, sean literarias-y que insiste en la importancia de estos cambios. Tanto en su narrativa de creación biológica como en su historia de creación novelística, Liboy se interesa por las genealogías, pero estas líneas de filiación no son sencillas y directas, sino torcidas, complicadas, rotas y reinventadas. Si la creación del hijo biológico de Molina se realiza gracias a la tecnología embriológica, la historia de esta concepción y nacimiento muestra una idea de familia que desafía los modelos tradicionales. Al mismo tiempo, al presentar una historia de amor que nunca existió como tal y una relación filial que todavía no se ha realizado, la novela deforma tanto la formación tradicional de las relaciones familiares como la estructura novelística. En este ensayo, propongo trazar algunas de estas líneas de filiación textual, ya que es mediante estas deformaciones (tanto intencionales como accidentales) que la genealogía literaria y la genealogía familiar se relacionan. El texto de Liboy reconoce la deformación -y con ella la embriología - como algo fundamental a la historia y la identidad nacional. Al mismo tiempo, Liboy "deforma" la narrativa oficial de la literatura nacional, ya que sugiere que la ciencia ficción es un género fundamental -y hasta fundacional- para la literatura puertorriqueña. La manera en que Liboy emplea el concepto en sus historias

4 Como nota Freedman, "The future is crucial to science fiction not as a specific chronological register, but as a locus of radical alterity to the mundane status quo, which is thus estranged and historicized as the concrete past of potential future" (55). 
de creación sugiere que la deformación no es sinónimo del error o del fracaso sino que también puede señalar una nueva dirección.

\section{Desvíos TeXTUALES: LA DOBLE CONCEPCIÓN}

Desde la primera página, El Informe Cabrera se identifica como un texto que se preocupa por la cuestión de filiación a la vez que la complica. Las primeras páginas del texto consisten en una carta de Manuel Molina a su hijo, carta en que le dice que le dará "[...] un informe de los beneficiarios que yo quisiera que tomaras en consideración" (9). Esta declaración parece indicar que Molina le dará a su hijo un bosquejo de su árbol familiar, o por lo menos una introducción a los miembros más significativos de su familia. Sin embargo, la carta -y los documentos siguientes- revelan que la estructura de esta familia no es tan sencilla. La primera persona "importante" que nombra Molina es su abuela, que sabe algo de la embriogenia y le explica a Molina que a pesar de la historia de deformaciones en la familia podría llegar a ser padre gracias a una muchacha que "[...] sería el vaso que permitiría tu nacimiento en caso de que los tiempos fueran adversos a personas como nosotros" (10). Cuando resulta que esta muchacha no va a poder ser la madre debido a las condiciones contaminadas en que vive (una mina oxidada), un maestro de literatura del pueblo cercano decide ofrecer "[...] certámenes literarios con el objeto de explorar si podíamos conseguir una madre voluntaria entre las muchachas que escriben [...]" (11). Estos son sólo los primeros eventos en la concepción de su hijo, y la historia ya se está complicando.

Como indica el relato del maestro de literatura, el tema de la concepción artificial del hijo de Manuel Molina se encuentra completamente entrelazada con el tema (y la práctica) de la literatura, de forma que la exploración de estos dos temas en el libro se enredan uno con el otro. La "serie de documentos" que Molina le ofrece a su hijo junto con su carta no consiste en documentos oficiales o textos científicos sino en la propuesta para una novela -también llamada El Informe Cabrera-junto con la aparente correspondencia de Molina con varios escritores (muchos de ellos poetas) y críticos literarios. Molina escribe al final de su "propuesta de novela", "Les invito a comentar este proyecto que tengo en mente, ya que en breve comenzaré la redacción del mismo [...]" (13), implicando que la correspondencia que sigue es su lado de un diálogo fructífero sobre no sólo el tema de la embriología y la concepción artificial sino también sobre la construcción de la novela misma. Dado ese marco metatextual, resulta difícil (por no decir imposible) saber si algunas de las anécdotas que supuestamente relatan partes de la historia de la concepción del hijo son verdaderas, o si todas forman parte de una ficción escrita o inventada por Liboy.

En este ambiente de "lo real múltiple", hasta los detalles se confunden. En la "propuesta de novela" Molina dice que su novela va a reunir "[...] una serie de 
documentos históricos que atestiguan la posibilidad de que un niño pepiniano ${ }^{5}$ naciera cien años después de la muerte de sus padres biológicos [...]" (13). Si la novela es ficción, ¿cómo puede ser también un foro para presentar documentos históricos? Y si los documentos sólo existen para atestiguar "la posibilidad" de hacer nacer un niño de esa manera, ¿es que realmente le nace (o nacerá) un niño a Molina, o no? El narrador del relato "El tocadiscos" dice, "Yo también soy un hijo póstumo" (45); si eso es cierto, parecería indicar que es bien posible hacer nacer un hijo de esa manera, porque el padre (Molina) es un ejemplo previo. En un fragmento hacia el final del libro titulado "Explicación a mi insistencia", explica que ha buscado en la embriología una explicación de "[...] por qué no podía vivir con [su] hijo" (74). No queda claro si está hablando de un hijo que está por nacer (y que no ha podido nacer todavía por causa de deformaciones genéticas) o si está hablando de un niño que ya existe.

Para complicar la línea entre la vida de Molina y lo que escribe, algunos de los relatos que aparecen en la novela tienen que ver con otros embriones y otros hijos creados de embriones en un laboratorio. Una anécdota del libro cuenta cómo el narrador (¿Molina?) viaja a un laboratorio en Santa Isabel con dos embriones, un embrión humano y otro de elefante. En el penúltimo "relato" del libro, titulado "Sonia”, el narrador hace (¿otro?) viaje a Santa Isabel en busca de un embrión perdido, ya que "[le] decían que el niño había nacido diez años después de la concepción" (78). "Parece" que este es un niño de otra familia que le ha encargado a Molina a que investigue el caso (79), pero queda poco claro si esta historia es algo que realmente le pasó a Molina o si es una exploración de algunos escenarios posibles relacionados con el tema de la embriología y la concepción.

\section{LA EMBRIOLOGÍA NUESTRA}

Si hay un hilo central que une la vida de Molina con su investigación/proyecto literario, es la embriología, tanto la ciencia genética como la embriología como metáfora. Como el narrador mismo admite, "[...] no puedo hacer otra cosa que comparar las ideas de las embriologías científicas con las ideas literarias" (75). Desde un punto de vista biológico, Molina parece necesitar la ayuda de la embriología para tener niños. Aunque nunca se describe a sí mismo físicamente ni explica exactamente por qué decide recurrir a la embriología para que nazca su hijo en el futuro, hace referencia al hecho de que hay muchas deformaciones en su familia y describe la teratología como "[...] la rama de la embriogenia que se ocupa de nosotros" (11). Observa que "Efectivamente vivimos en una época de escasos valores morales, y cabía esperar que se nos rechazase por nuestros defectos físicos [...]" (10), declaración que implica que las deformaciones que afectan

El adjetivo pepiniano se refiere a alguien de la región puertorriqueña de San Sebastián, ya que este municipio se conocía anteriormente como San Sebastián del Pepino. 
a su familia son de alguna manera físicamente notables. En el caso de su hijo, no es que quiera que la embriología le ayude a producir un superhombre sino que quiere que le dé la oportunidad de tener un hijo normal y saludable.

Sin embargo, la carta de Molina se desvía casi inmediatamente de cualquier intento de presentar una perspectiva clara, sea de la familia Molina, sea de la embriología misma. Molina le declara a su hijo, "La experiencia te demostrará que tu destino es más importante que tu sangre, y que la embriología verdadera tiene mucho que ver con esos destinatarios que no participan directamente de tu nacimiento, pero que son como tus padres silentes, sombras vivas que te rodean y acompañan más allá de la experiencia visible" (9-10). Con esta aseveración, Molina parece rechazar los principios biológicos de la genealogía (y de ahí la embriología); plantea que nuestra formación tiene menos que ver con la sangre, las células y el código genético que con la formación psíquica de un individuo, con el desarrollo emocional/espiritual que ocurre gracias a personas que no son necesariamente los padres biológicos. El embrión del que habla, de hecho, no parece ser un embrión de células sino una especie de embrión espiritual. La descripción de "sombras vivas que te rodean" parece más apta para espíritus que para seres de carne y hueso, ya que la ciencia generalmente trata lo que podemos percibir, no lo que yace "más allá" de lo visible.

Queda claro desde este momento que estamos frente a unas ideas divergentes de la embriología. Molina mismo identifica dos: la primera, que se podría nombrar la "clásicamente" biológica, describe cómo "[...] la embriología de Dolly, el clon de oveja del que tanto se habló en los noventa, por el hecho de que haya participación de varias madres [...]" (15). Este tipo de embriología se relaciona con el escritor y su hijo, en el sentido en que el hijo nacerá gracias a la participación de más de una madre, y que por eso ellos podrían ser vistos como "curiosidades" como la oveja Dolly (11). Sin embargo, gracias a los problemas teratológicos en su génetica familiar, el narrador tendrá que recurrir a la embriología biológica si quiere tener un hijo.

La segunda especie de embriología, tanto como la descripción anterior de los seres queridos, es una ciencia "autóctona". Molina observa que su abuela paterna le hablaba de la práctica de la embriología junto con el espiritismo, y que consideraba "[...] que la embriología insular era una costumbre, un dato folklórico de nuestra isla" (14). Describe la ciencia como una práctica cultural local, algo no necesariamente visible pero común y corriente. Más tarde habla de sus labores de encontrar textos puertorriqueños sobre la embriología, ya que "[t]odo lo que se hablaba de embriología en aquel entonces tenía el carácter de una cultura oral" (42). Si la embriología “clásica" produce curiosidades como la oveja Dolly, la embriología isleña parece ser una cosa tan integrada a la sociedad que no es necesariamente reconocida como tal. Gracias a su "oralidad” y su conexión con el espiritismo, funciona también como una práctica "secreta" visible pero no reconocida. Su carácter oral contrasta con la búsqueda literaria (textual) que emprende Molina al tratar de localizar ejemplos de la embriología en la literatura de la isla. 
Al identificar una embriología local, vinculada a las tradiciones de la isla, Liboy hace una referencia - paródica, distorsionada-al papel de la planificación familiar en la historia de Puerto Rico. Al establecer una relación colonial con Puerto Rico al final de la Guerra Hispanoamericana, los líderes coloniales estadounidenses identificaron a los habitantes de las isla como wayward and unruly e iniciaron una serie de mecanismos de control políticos y sociales (Santiago-Valles 20). Además de dirigir la productividad y el trabajo, estos mecanismos también intentaban controlar y subyugar a los cuerpos puertorriqueños, racializados como "inferiores" y vistos como dotados de una sexualidad “excesiva” o "divergente" (Briggs 75). Como plantea Laura Briggs, desde el final del siglo XIX, "[...] reproduction and sexuality have defined the difference that makes colonialism in Puerto Rico possible and necessary" (4). Estas prácticas incluían intentos de controlar la planificación familiar y la tasa de natalidad a través de métodos anticonceptivos: Briggs muestra cómo la fertilidad femenina y la planificación familiar llegaron a ser los temas discutidos en un debate político sobre el nacionalismo, el capitalismo, la pobreza y la modernidad (75). Uno de los métodos anticonceptivos más politizados era la esterilización; una ley en 1937 creó un Consejo de Eugenesia, y en los treinta años siguientes, los hospitales de la isla hicieron un número record de esterilizaciones (Briggs 148-50). Aunque algunos políticos puertorriqueños apoyaban la práctica, y muchas de las esterilizaciones fueron "voluntarias", otros nacionalistas denunciaban la práctica como una estrategia de control norteamericana. Parte de la controversia sobre los métodos anticonceptivos tenía que ver precisamente con su papel en las políticas coloniales de la isla. Había rumores de una campaña secreta estadounidense de acabar con la población puertorriqueña a través de la esterilización.

El Informe Cabrera da vuelta a las condiciones controversiales de la situación histórica de la planificación familiar; si los poderes políticos -tanto externos como internos- luchaban por controlar las condiciones de la fertilidad puertorriqueña (y los cuerpos de las mujeres puertorriqueñas), la novela de Liboy define la embriología -y con ella la planificación familiar- como una práctica cultural autóctona, dotada de matices (como el espiritismo) que no tienen ninguna conexión con el exterior. En vez de presentarnos con una tasa de natalidad nacional vista como demasiada alta, la isla del texto de Liboy nos presenta (irónicamente) con una familia que tiene dificultades en reproducirse. El texto también reemplaza el secreto de la esterilizaciones forzadas con un laboratorio "secreto" en San Sebastián, el municipio en la parte occidental de la isla donde el hijo de Manuel Molina será engendrado. Que el laboratorio ocupe el sitio de la Central Soller, anteriormente una de las haciendas azucareras más grandes de la isla, no es una casualidad: lo que era el centro de poder (a través de la producción agrícula-industrial) en otro momento histórico será re-imaginado en el escenario de la novela como un centro de investigación científica. La embriología en este escenario no es una ciencia importada sino autóctona. 
Además de jugar con las controversias sobre la reproducción puertorriqueña, la situación de Molina hace una referencia indirecta a los problemas medioambientales que resultan (en parte) del colonialismo estadounidense. Puerto Rico lleva mucho tiempo como un centro de producción para la industria farmacéutica estadounidense. A pesar de los muchos beneficios económicos, esta industria es uno de los principales contribuyentes a la contaminación medioambiental de la isla. ${ }^{6}$ (Otra fuente de contaminación sería el ejército estadounidense, que por muchos años usó la isla de Vieques como un sitio de experimentación para bombas militares). El texto de Liboy no habla directamente de las fuentes de contaminación, pero el hecho de que la primera prospectiva "madre" para el hijo de Molina no puede tener hijos porque vive en una mina oxidada indica que la contaminación medioambiental es un factor presente (aunque no nombrado) en la historia. La novela presenta un escenario donde la embriología es una necesidad para gente en la situación de Molina.

Ala vezque el texto de Liboy propone la existencia de una embriología puertorriqueña "nacional", la novela también se enfoca en la embriología como una ciencia literaria. Molina explica que su texto se llama "El Informe Cabrera" en honor a una familia puertorriqueña que demostraba un interés temprano en la embriología, no como parte de la eugenesia u otras pseudo-ciencias sino "[... ] liberada de los errores de la embriología más conservadora" (9). Estamos frente a dos genealogías, la de Manuel Molina y su hijo nonato, y la de la familia Cabrera, estirpe de escritores y de científicos. A través de su identidad binaria, la familia Cabrera conectará la biología con la literatura: el narrador identifica el segundo tipo de embriología, la autóctona, como una embriología literaria. Gran parte del resto del libro consistirá en establecer una bibliografía de textos y escritores que tratan la embriología. Este corpus, tanto como la embriología en la novela, será una combinación de elementos de la vida real y detalles ficticios e inventados. Resulta que estos son también los escritores y textos que Liboy reconocerá como ejemplos de una ciencia ficción puertorriqueña. Para entender lo que Liboy quiere hacer con esta genealogía, será necesario hacer un breve recorrido por este corpus.

\section{DE PADRES E HIJOS LITERARIOS: LECTURAS ALTERNAS}

El primer escritor que Molina (Liboy) reconoce como un autor que ha tratado el tema de la embriología es Pablo Morales Cabrera (1866-1933), periodista que llegó a ser más conocido como cuentista, y la "raíz" del árbol genealógico de la familia Cabrera. A pesar de ser un escritor conocido dentro de la cuentística puertorriqueña, la imagen que Molina/Liboy pinta de Pablo Morales Cabrera va cambiando a lo largo del texto.

${ }^{6}$ Para más sobre esa historia -y los efectos medioambientales, ver Alexa S. Dietrich, The Drug Company Next Door. 
Molina observa en la primera página de la carta (y del libro) que fue Morales Cabrera el que "[...] por primera vez, y sin poder hacer otra cosa, expuso claramente todos los prejuicios y los mitos relacionados a la embriogenia" (9). En la siguiente sección del libro, sin embargo, explica que Morales Cabrera publicó varios cuentos "con el tema de la embriología como eje central" (12) en el siglo XIX, hecho falso si estamos hablando de la vida real del escritor, ya que sus Cuentos populares no salieron a la luz hasta 1914. ¿Por qué ese cambio -aparentemente trivial-de fechas?

La caracterización de Morales Cabrera como el primer cuentista de la embriología es aún más interesante si miramos los cuentos que Molina identifica como historias que tratan la embriología. Hay dos: "El kleptómano" y "Las camándulas", las cuales se incluyen en la colección Cuentos populares. En "El kleptómano", Indalecio, un hombre de familia humilde, está haciendo un esfuerzo por subir en la escala social cuando se enamora de una viuda atractiva. Cuando la viuda parece estar embarazada y después se adelgaza rápidamente, todos en el pueblo no sólo sospechan que Indalecio es el padre sino que también concluyen que le ha ayudado a la viuda a matar al bebé. Algunos habitantes del pueblo encuentran a Indalecio enterrando algo en lo que parece ser una fosa, asumen que es el cadáver del recién nacido y arrestan a Indalecio. Al final, sin embargo, descubren que ese "cadáver" no es nada más que un muñeco que Indalecio -el cleptómano del título- ha robado. El muchacho es dejado en libertad, pero sus intenciones de ocupar una posición alta en el pueblo son frustradas, gracias a sus tendencias cleptómanas.

En términos de trama, "El kleptómano" no parece cumplir con lo que un lector imaginaría de una historia sobre la embriología, ni de un texto de ciencia ficción. La única conexión posible con esa ciencia en el texto aparece en la descripción de Indalecio: “¡Pobre Indalecio! Creo que él no tenía madre; nació de un esporo y creció como un hongo, más, si un botánico lo hubiese catalogado, lo incluye entre las especies venenosas" (161). Se vuelve a repetir esta descripción al final del ensayo, añadiendo que Indalecio "ya estaba clasificado, como parasitario" (164). Si se lee el texto como un texto de tono realista, parece claro que la descripción del protagonista es una metáfora: Indalecio está tan solo en el mundo que es como si se hubiera nacido sin una madre (viva o presente) y sin parientes que lo puedan ayudar. Esta situación es tal vez la causa de su cleptomanía y de su inhabilidad de encontrar un trabajo apropiado. Liboy, sin embargo, quiere leer esta cita en un sentido literal: Indalecio no es el producto de la unión de dos seres humanos sino que ha nacido gracias a otros métodos científicos. Siendo esto el caso, su condición como cleptómano, "producto morboso de la degeneración humana" no tiene nada que ver con su raza ni su vida familiar y todo que ver con la manera (científica) en que fue engendrado, una manera que literalmente no es humano. Seo-Young Chu explica que en la ciencia ficción, "Occurences of figurative language in SF texts and contexts have an interesting tendency to elicit literal interpretation almost as a matter of course" [...] (10). 
En el caso del cuento de Morales Cabrera, leer la descripción metafórica de Indalecio como si fuera literal produce un significado completamente diferente. Indalecio no actúa de la manera en que actúa por razones psicológicas sino porque literalmente ha sido engendrado de esa manera, porque es un ser completamente extraño.

Al identificar un texto como "El kleptómano" como un texto de ciencia ficción, Liboy no reconoce una manera particular de escribir sino que propone una manera diferente de leer. Como Molina observa, "Una novela explícita de embriología no funcionaría. El juego es descubrir textos donde el sujeto oculto no se haya desarrollado" (16). Leer instituyendo un alto nivel de mímesis - desde un punto de vista "ciencia ficcional" - transforma el significado de ciertos textos, haciendo salir a la luz toda una serie de preocupaciones y tendencias no antes reconocidas. Molina después aplica este método de leer a José Martí y plantea que en la poesía del cubano “[...] las palabras de la libertad, las palabras éticas, están desnaturalizadas por la experiencia de una forma poética" (32). Molina encuentra en "El padre suizo", poema basado en el caso de un emigrante suizo en Arkansas que mata a sus tres hijos y después se suicida la mención de "estrellas luminosas" en el texto es una clara referencia al espacio y plantea que en vez de leer la referencia a los astros en el poema (que "guiarán" al padre muerto) como un ejemplo de "la astrología judiciaria" (como signos que acompañan el padre), se debe leerlos "como límite o condición” (34). El espacio en Martí expresa una "oscilación” entre una libertad que "se afirma contra lo venial, lo doméstico" y la conciencia de que a esa libertad "[...] tampoco una exterioridad conviene, que se deshace en la ciudad y el espacio" (34). Si leer las metáforas embriológicas en Morales Cabrera como literales produce un "extrañamiento cognitivo", leer la referencia al espacio como literal lleva a niveles de interpretación que parecen hasta "menos" literales y más metafóricos.

Si como declara Molina, "Se puede decir que todas las épocas tienen su novela sobre embriología y en cada época la disciplina se relaciona al tema más importante del momento en que se escribe" (17), lo que más parece interesarle a Liboy es la serie de preocupaciones que se revela en la escritura de miembros de su propia generación. Leer desde una perspectiva altamente mimética ("ciencia ficcional") le permite a Liboy crear un nuevo canon de textos clásicos, pero también le ayuda a crear una comunidad de contemporáneos, de establecer un diálogo con otros escritores puertorriqueños "raros". Las muchas "cartas" que aparecen en la novela reflejan una correspondencia activa entre Molina (Liboy) y escritores puertorriqueños contemporáneos como Edgar Ramírez Mella, Diego Deni (Pedro Cabiya) ${ }^{7}$ Yara Liceaga, Kattia Chico, y Carlos López Dzur. Si en el siglo XIX y a principios del siglo XX la embriología estaba conectada

\footnotetext{
7 Pedro Cabiya (1971), un escritor puertorriqueño que radica desde hace varios años en la República Dominicana, publicó su primer libro Historias tremendas (1999) bajo el pseudónimo Diego Deni, que es el nombre que usa en El Informe Cabrera.
} 
con ideas de la degeneración y la eugenesia, la generación de los años noventa “[ ...] no maneja el tema de la embriología negativamente" (17). Estos escritores forman una comunidad para Molina (Liboy) porque su escritura posibilita una manera particular de leer o porque ellos también están leyendo desde un punto de vista que no sigue los contornos del realismo más esperado.

Las menciones a la embriología que Molina encuentra llegan a ser una especie de código para esa manera "deformada" de leer, un código que indica las posibilidades de un texto particular -o la obra de un escritor particular-para ser leída desde el lente "literal" de la ciencia ficción. En una carta a la escritora Yara Liceaga, por ejemplo, Molina caracteriza la embriología en su obra como "una embriología forense" (36). Para ilustrar este concepto describe brevemente un cuento de Liceaga: "La protagonista sostiene relaciones amorosas con un andaluz y enseguida visita un cementerio importante. La idea de que hay un hijo enterrado de ambos no se dice, pues el cuento es muy sugerente, no directamente del tema" (36-7). Aquí la idea de la "embriología forense" tiene varios sentidos posibles. Que el hijo puede estar muerto, que el cuento tiene lugar en un cementerio parece indicar que cualquier trabajo científico tendrá que investigar cosas muertas. Sin embargo, como la ciencia forense descubre historias borradas usando la evidencia encontrada en un cadáver, llamar la técnica de Liceaga "forense" sugiere que ella ha encontrado una manera nueva de comunicar con el material ya "muerto", que tiene una habilidad de reconstruir un escenario escondido. Si se trata de hacer una lectura de "ciencia ficción" de ese material, esta "lectura forense" consistiría en crear un escenario en que son posibles varios niveles de "intensidad" del realismo (para adaptar el concepto de $\mathrm{Chu}$ ).

A veces la lectura de Liboy consiste en una deformación hasta de estos textos "raros". Sería imposible trazar un canon de la ciencia ficción puertorriqueña y no decir nada de Rafael Acevedo y su novela Exquisito cadáver (2003). Como Molina/Liboy admite, manipulando ligeramente el título del libro, "[U]na novela de ficción científica no se había escrito conscientemente hasta Cadáver exquisito" (29). La novela tiene lugar en un San Juan distópico y futurista; el protagonista de la novela es un detective que se enamora de una mujer que podría ser un cyborg. A través de su relación con esta mujer, comienza a cuestionar el sistema cibernético totalitario que controla la isla. Sin embargo, el texto de Acevedo presenta un problema para el proyecto de Molina: es una novela de matices cyberpunk, que no trata el tema de la embriología.

Liboy no puede conectar este enfoque cibernético de la novela de Acevedo a la embriología, pero sí los puede conectar a través de la analogía. Comienza hablando de las ideas pseudo-científicas racistas de escritores decimonónicos como Edouard Balbiani, y explica que llegaban a esas teorías a través de las falsas analogías; por ejemplo, intentaban explicar la sexualidad de las mujeres negras usando lo que se sabía de la sexualidad de las moscas. Sucede que la falsa analogía es también "[...] uno de los 
pilares conceptuales de la ciencia ficción de cualquier país" (29), así que las ideas de estos escritores, a pesar de ser escritas con las intenciones más científicas, llegan a ocupar el registro de la ciencia ficción. El texto de Acevedo, en cambio, no habla de embriología pero llega a emplear "intencionalmente" la misma estrategia (de ciencia ficción) que usaban estos primeros investigadores de una embriología pseudo-científica; usa "[...] el medio cultural más interesado, que era la neurología que se estudia en Puerto Rico" (31) para hablar de cómo los medios de control operan en la sociedad puertorriqueña. Molina/Liboy, sin embargo, ofrece otra manera de leer el texto: "La novela de Acevedo se ha salvado porque los personajes del texto somos sus amigos disfrazados de asesinos y policias" (31). Es Liboy aquí que crea una falsa analogía, o por lo menos, desvía la expectativa del lector de poder establecer una analogía exacta entre la escritura de los embriólogos decimonónicos y la de Acevedo. El texto de Acevedo se vende como ciencia ficción, y puede ser leído como tal, cuando realmente está hablando de una manera de leer la sociedad y el control social. Según otra lectura, sin embargo, es un chiste elaborado sobre una comunidad literaria particular.

\section{LA HISTORIA DESDE EL FUTURO}

A lo largo de El Informe Cabrera, la embriología funciona como el sendero de migas que seguimos en nuestro intento de no perdernos en el bosque del libro. Es la ciencia que le ayudará a Manuel Molina a tener un hijo, y un leitmotiv en un posible canon de la ciencia ficción puertorriqueña. Sin embargo, en una carta al principio del texto, Liboy revela que hasta el enfoque en la embriología es en sí un desvío de la intención central del libro:

El meollo del asunto no es en sí la maternidad compartida, ni el hecho feudal de quién hereda o no el código del viajero, sino la proyección temporal del texto.... Mi novela estudia la conducta fenotípica (las imitaciones, los plagios, los carnavales, las parodias), como elementos que de alguna manera guían la proyección temporal del viajero niño. (26-7)

Esta cita ilustra el argumento de Freedman sobre la importancia del futuro como un lugar desde donde llegan a una nueva perspectiva sobre el pasado y el presente. Para Liboy, las ficciones del presente, los pequeños desvíos cotidianos y posibles crean el espacio para este niño futuro. Por eso insiste tanto en compilar su genealogía de textos de ciencia ficción: sin las posibilidades descriptivas expandidas de este género, otras ficciones científicas y otras analogías aún más intensas no serán posibles en el futuro. Eso no es decir que Liboy tiene una propuesta, un camino particular en mente; Quintero Herencia dice de la escritura de los "raros": "La singularidad de estos textos radicaría en su negativa a reconfigurar alguna jerarquía moral, subjetiva o espacial que de cuenta 
del descampado o el desierto significante donde se ensamblan sus escrituras" (158). Sin embargo, estos textos sí pueden comenzar a poblar el descampado. Tal como aparece en El Informe Cabrera, la ciencia ficción para Pepe Liboy ofrece una salida de este tipo. Si la realidad de Puerto Rico (tanto como su historia como colonia estadounidense) está llena de distorsiones, alterar esas alteraciones puede resultar en la creación de una nueva solución, una nueva realidad, tanto dentro de la realidad de la isla como dentro de su historia literaria.

\section{BibLIOGRAFÍA}

Briggs, Laura. Reproducing Empire: Race, Sex, Science and U.S. Imperialism in Puerto Rico. Berkeley: U of California P, 2002.

Chu, Seo-Young. Do Metaphors Dream of Literal Sleep? A Science Fictional Theory of Representation. Cambridge, MA: Harvard UP, 2011.

Dietrich, Alexa S. The Drug Company Next Door: Pollution, Jobs, and Community Health in Puerto Rico. Nueva York: New York UP, 2013.

Duchesne Winter, Juan. "Noticias de un país que desaparece, 'raros' puertorriqueños de hoy". América Latina Hoy 58 (2011): 31-50.

Freedman, Carl. Critical Theory and Science Fiction. Middletown, CT: Wesleyan U P, 2000.

Gledhill, Christine. "Rethinking Genre." Reinventing Film Studies. Christine Gledhill y Linda Williams, eds. Londres: Arnold, 2000. 221-43.

Liboy Erba, José. Cada vez te despides mejor. San Juan: Isla Negra, 2004. El informe Cabrera. Lajas, Puerto Rico: Concepción 8, 2009.

Morales Cabrera, Pablo. Cuentos populares. San Juan: Baldrich, 1939.

Quintero Herencia, Juan Carlos. "Extranjería, comunidad y escucha: escenas contemporáneas de autoridad literaria puertorriqueña". Centro Journal 22/2 (2010): 151-171.

Santiago-Valles, Kelvin A. "Subject People" and Colonial Discourses: Economic Transformation and Social Disorder in Puerto Rico, 1898-1947. Albany: SUNY P, 1994.

Suvin, Darko. "On the Poetics of the Science Fiction Genre." College English 34/3 (1972): 372-82. 